\title{
Oral Dantrolene Causing a Decrease in Vital Capacity, Recurrent Lung Collapse, and Respiratory Failure in a Spinal Cord Injury Patient: A Case Report
}

\section{Kevin C. Proud ${ }^{1 *}$, Rana $\mathrm{Hejal}^{2}$ and Hugo Montenegro ${ }^{2,3}$}

${ }^{1}$ University of Texas Health Science Center at San Antonio, Texas, USA

${ }^{2}$ University Hospitals Cleveland Medical Center, Case Western Reserve University, Cleveland, Ohio

${ }^{3}$ Louis Stokes Cleveland VA Medical Center Cleveland, Ohio

\begin{abstract}
Introduction: Dantrolene is a muscle relaxant used to treat malignant hyperthermia as well as muscle spasms in spinal cord injury patients. It is known to cause a decrease in skeletal muscle strength in humans, and inhibit diaphragm muscle strength in hamsters. There is at least one other case report of dantrolene leading to respiratory failure in a spinal cord injury patient. Here we present a case of a patient developing poor mucus clearance, lobar collapse and respiratoy failure after being started on dantrolene, and trend vital capacity and negative inspiratory force while titrating off dantrolene.
\end{abstract}

Case: $47 \mathrm{y} / \mathrm{o}$ male with history of motor vehicle accident resulting in tetraplegia 19 months prior to admission, presented with pneumonia for the first time since his accident. He also experienced recurrent episodes of bilateral lung collapse, respiratory failure and was found to have significant respiratory muscle weakness. He had been started on dantrolene approximately six weeks prior to admission. After stopping dantrolene his clinical status, his vital capacity and negative inspiratory force (NIF) dramatically improved. He was then able to clear his secretions, and had no further episodes of lung collapse.

Discussion: Our case is only the second in literature of dantrolene causing respiratory failure in a spinal cord patient; and is the first to trend daily vital capacity and NIF while the patient is being tapered off the dantrolene. Our case documented a dramatic improvement NIF and FVC after stopping dantrolene. Our case demonstrates the potential and avoidable harm of using dantrolene in a group of patients for which is commonly prescribed.

Keywords: Respiratory muscle; Weakness; Dantrolene; Spinal cord injury; Pharmacy; Neurology

\section{Case Presentation}

A 47 y/o male with a history of C6 tetraplegia due to a motor vehicle accident 19 months prior presented to an outside hospital with complaints of fever, shortness of breath, and productive cough, and a CT scan that was consistent with bilateral pneumonia and atelectasis. Immediately after his accident he had a tracheostomy, however was subsequently deccanulated and was without respiratory support for greater than 17 months. His wife noted that since being since being started on medication for muscle spasms he had increased difficulty clearing his respiratory secretions.

At the outside hospital vital signs were notable for a temperature of $101.2^{\circ} \mathrm{F}$ and oxygen saturation of $89 \%$ on room air. He was started on broad spectrum antibiotics and subsequently transferred to the spinal cord unit of our hospital for further care. Four days into his admission, he experienced an acute worsening of hypoxia and was transferred to the MICU. There he was noted to have near complete collapse of his left lung (Figure 1). Despite aggressive chest physiotherapy and suctioning he was ultimately intubated.

Sputum cultures confirmed pseudomonas pneumonia and he was treated with appropriate antibiotics and extubated nine days later. Two days after extubation he again desaturated and chest $\mathrm{x}$-ray revealed near complete collapse of his contra-lateral lung (right lung) (Figure 1). He was treated with suctioning and Intermittent Positive Pressure Breathing (IPPB) with some improvement. He was subsequently treated with nightly NIPPV due to mild $\mathrm{CO}_{2}$ retention and frequent recurrences of various areas of lobar collapse.

Tracheostomy was discussed with the patient's wife, who was hesitant and re-emphasized that he had not had issues with respiratory muscle weakness or trouble clearing secretions until a few weeks prior to this admission, and noted that it correlated with the patients discharge from spinal cord unit for muscle spasms six weeks prior to his current admission. During that hospitalization dantrolene was started and was titrated up to $50 \mathrm{mg}$ po four times per day.

The MICU team then suspected dantrolene as a possible culprit for his respiratory muscle weakness, and started to check daily Forced Vital Capacity (FVC) and negative inspiratory force (NIF). Dantrolene was then tapered from $50 \mathrm{mg}$ po QID to $50 \mathrm{mg}$ po BID to $25 \mathrm{mg}$ po BID to $25 \mathrm{mg}$ po daily, and then stopped completely. His initial VC was 280 and NIF was -10 . Both the FVC and NIF gradually improved during the taper (Figures 2 and 3). His episodes of lung collapse and desaturations resolved. Eight days after starting the dantrolene taper, he was transferred out of the ICU, where he continued to do well without further pulmonary issues and his FVC gradually improved to as high of $2.6 \mathrm{~L}$ and NIF consistently more negative than -60 (the maximal reading on our portable PFT machine). While he did have some variation in the daily FVC off dantrolene as seen on days 11 and 12 in Figure 2, the mean FVC off dantrolene s nearly double that compared to when he was on the medication (Figure 4).

\section{Discussion}

Dantrolene is a muscle spasmolytic that was developed in 1967 [1] It works primarily on skeletal muscle, but does have a weak effect on

*Corresponding author: Kevin C. Proud, University of Texas Health Science Center at San Antonio, Texas, USA, Tel: 210743 0933; E-mail: Proud@uthscsa.edu

Received December 29, 2016; Accepted January 19, 2017; Published January 24, 2017

Citation: Proud KC, Hejal R, Montenegro H (2017) Oral Dantrolene Causing a Decrease in Vital Capacity, Recurrent Lung Collapse, and Respiratory Failure in a Spinal Cord Injury Patient: A Case Report. Lung Dis Treat 3: 117. doi:10.4172/2472 1018.1000117

Copyright: $\odot 2017$ Proud KC, et al. This is an open-access article distributed under the terms of the Creative Commons Attribution License, which permits unrestricted use, distribution, and reproduction in any medium, provided the original author and source are credited. 


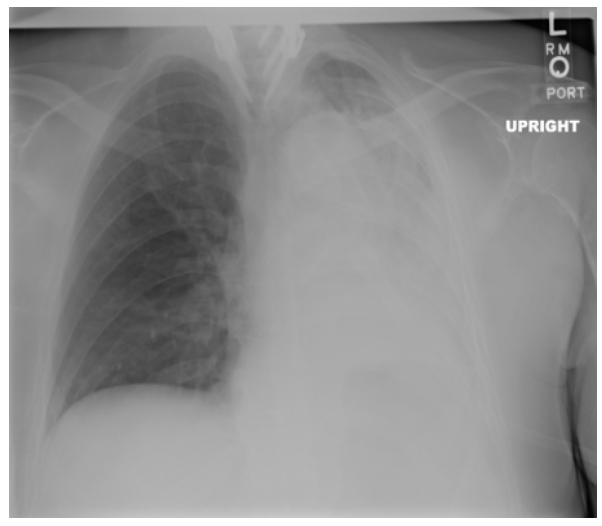

a)

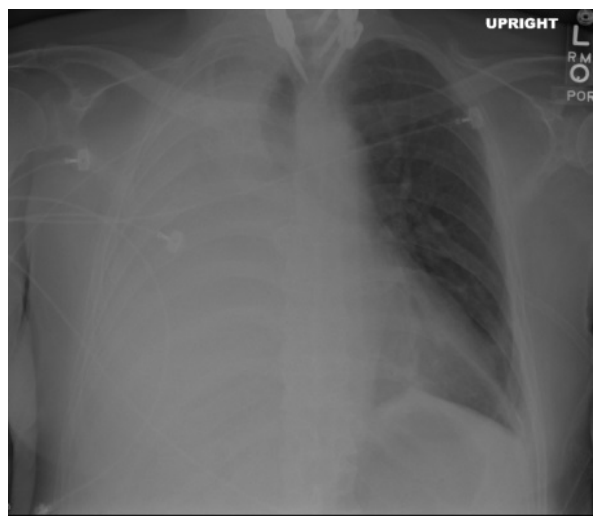

b)

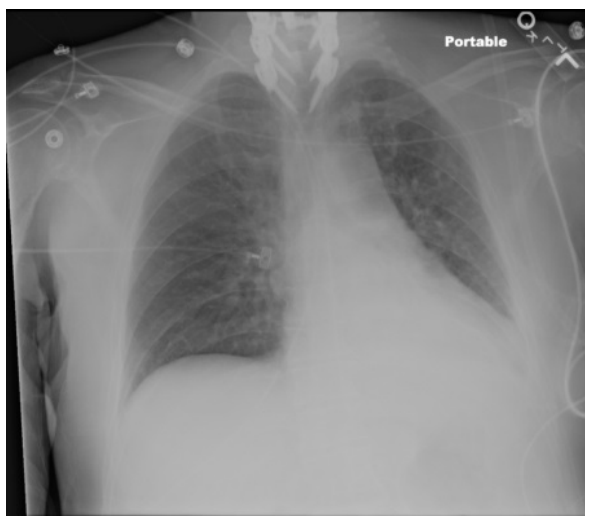

c)

Figure 1: a) ICU day 1 (on dantrolone), b) ICU day 14 (on dantrolene), and C) ICU day 24 (off dantrolene)

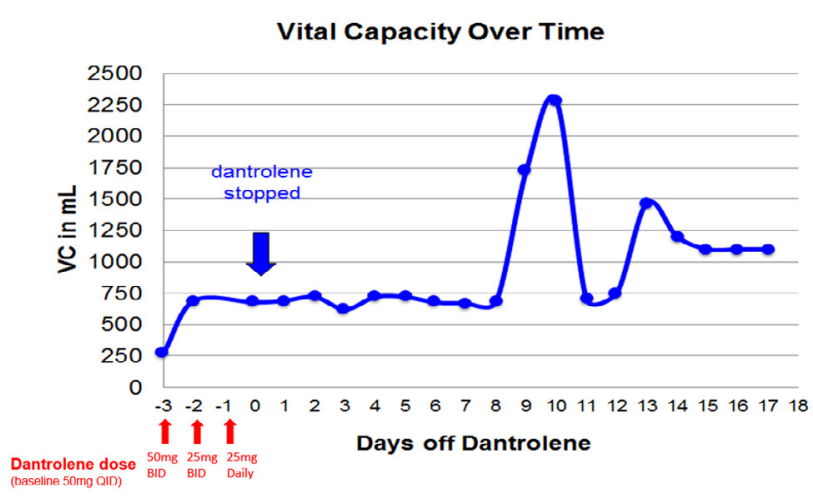

Figure 2: Graph showing the vital capacity of Dantrolene over time.

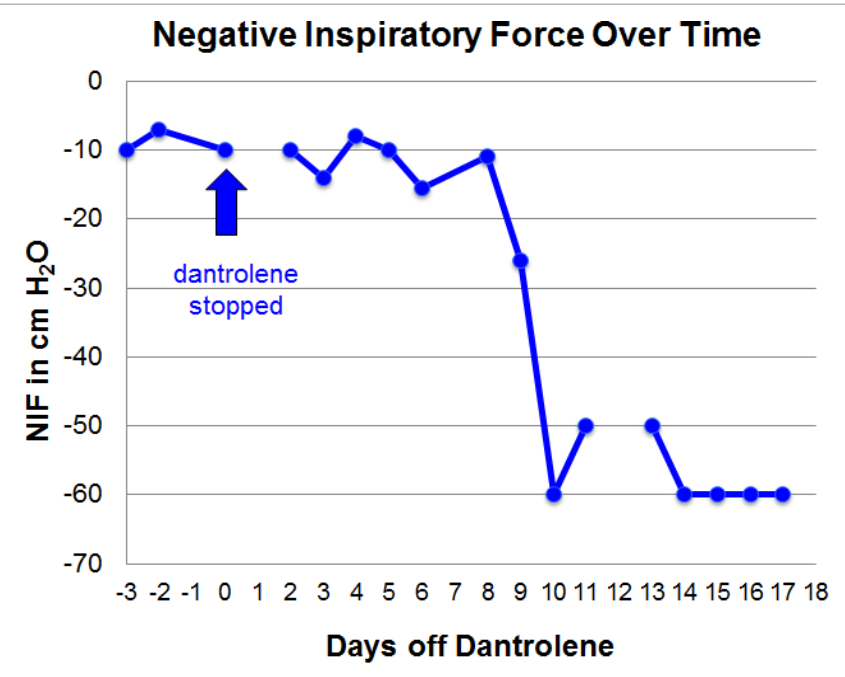

Figure 3: Graph between NIF in centimetre $\mathrm{H}_{2} \mathrm{O}$ and days off Dantrolene.

smooth muscle and no effect on myocardial muscle contraction [2]. While dantrolene's mechanism of action is not completely understood, it is well established that it binds to the Ryanodine Receptor (RYR) channel located on the sarcoplasmic reticulum (SR) and thereby prevents the release of calcium from the SR into the cytoplasm $[1,3]$.
This process seems to be dependent on the presence of a RYR activity regulator calmodulin [4]. The paucity of calcium limits excitationcontraction coupling in the muscle fibers, and it has been shown not to have any effect on the action potential of the neuromuscular junction. Dantrolene cannot cause complete paralysis even in supra-therapeutic doses. The recommended starting oral dose is $25 \mathrm{mg}$ po daily, and maximum oral dose is $100 \mathrm{mg}$ po four times per day. Approximately one-third of the oral dose is absorbed.

Dantrolene was first used to treat skeletal muscle spasticity, and continues to be commonly used to treat patients with spasms following cerebral vascular accidents or spinal cord injuries. Its use has also expanded to be first line treatment for malignant hyperthermia. It has also been used for neuroleptic malignant syndrome, heat stroke, and ecstasy (3,4-methylenedioxymethanamphetamine) intoxication [1]. More recently dantrolene is being evaluated as a possible antiarrhythmic, especially in the setting of ventricular fibrillation $[5,6]$.

Dantrolene's effect on the respiratory system thus far is poorly classified, and likely represents an underappreciated source of respiratory problems. There are multiple case reports describing pleural effusions associated with long-term use. More concerning, however is the possibility of respiratory failure in susceptible patients. In a review of 368 cases of malignant hyperthermia treated with dantrolene, Brandom et al, reported a 3.8\% rate of respiratory failure. The exact circumstances of the respiratory failure in each of those cases were not described, though 1 had known myopathy prior to the event [7]. There is at least one other case report of severe respiratory failure in a spinal cord patient who received oral dantrolene [8]. In that case report, the patient's dose and the elapsed time between titration of the medication, and onset of respiratory symptoms were similar to our case. Additionally $\mathrm{CO}_{2}$ retention was seen in both patients.

Flewellen et al., administered intravenous dantrolene to healthy volunteers and saw a mean decrease in grip strength of 42\% [9]. Peak Expiratory Flow (PEF) and Forced Vital Capacity (FVC) were also measured immediately following each dantrolene administration and up to $4 \mathrm{~h}$ after the last dose of dantrolene, but no significant change was seen. Contradictory to that, however, is the work done by Langeron et al., who evaluated the effect of dantrolene on diaphragm muscle of hamsters. A dose dependent negative inotropic effect was observed, resulting in a decrease in active inotropic force of $34 \%$ in normal hamsters, and $32 \%$ in myopathic hampters $[10,11]$. 


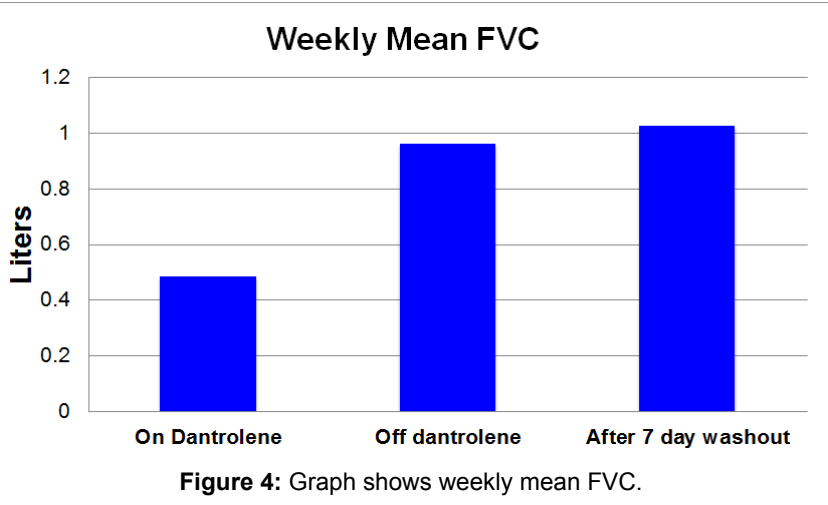

In our patient, FVC and NIF were measured daily starting on the day of the initiation of dantrolene taper. Despite some variability in daily FVC readings, our patient's FVC increased from a mean of 485 $\mathrm{ml}$ on dantrolene to a mean of $1020 \mathrm{~mL}$ after a 7 day washout period off dantrolene. Similarly, the NIF went from a mean of $-9.4 \mathrm{~cm} \mathrm{H}_{2} \mathrm{O}$ on dantrolene to a mean of $-34 \mathrm{~cm} \mathrm{H}_{2} \mathrm{O}$ following the washout period. This interpretation is somewhat limited as the device used to measure NIF could only read up to $-60 \mathrm{~cm}$ of water, which the patient reached on 11 out of his last 15 measurements. Additionally, as mentioned in the other case report, the patient was often drowsy when off the medication. Since being discharged, the patient has gone over 13 months without a recurrence of pneumonia or admission for hypercapnea since stopping the dantrolene.

\section{Conclusion}

We believe that dantrolene led to a worsening of this patient's respiratory muscle strength, which led to decreased clearance of secretions, resulting in pneumonia and multiple episodes of lung collapse and respiratory failure. Dantrolene may tip a patient that already has underlying respiratory muscle weakness into respiratory failure and therefore should be titrated with caution in such patients. Ideally, baseline pulmonary function tests should be done prior to starting and during the titration of oral dantrolene in spinal cord patients. Because this medication is currently a first line therapy for muscle spasms in this patient population, further investigation of its safety in this subgroup is likely necessary, but until then caution and close monitoring when using dantrolene for this indication is advised.

\section{References}

1. Krause T, Gerbershagen MU, Fiege W, and Wappler F (2004) Dantrolene-A review of its pharmacology, therapeutic use and new developments. Anaesthesia 59: 364-373.

2. Ellis KO, Butterfield JL, Wessels FL, Carpenter JF (1976) A comparison of skeletal, cardiac, and smooth muscle actions of dantrolene-a skeletal muscle relaxant. Arch Int Pharmacodyn Ther 224: 118-132.

3. Katzung B (ed.) (2004) Basic and Clinical Pharmacology ( $9^{\text {th }}$ edn.). McGrawHill, USA, pp: 444-445.

4. Oo Y, Gomez-Hurtado N, Walweel K, Van Helden D, Imtiaz M, et al (2015) Essential Role of Calmodulin in RyR Inhibition by Dantrolene. Mol Pharmmacol 88: 57-63.

5. Zamiri N, Masse S, Ramadeen A, Kusha M, Hu X, et al (2014) Dantrolene Improves Survival After Ventricular Fibrillation by Mitigating Imparted Calcium Handling in Animal Models. Circulation 129: 875-885.

6. Wiesmann T, Freitag D, Dersch W, Escbach D, Irqsusi M, et al (2017) Dantrolene versus amiodarone for cardiopulmonary resuscitation: a randomized doubleblinded experimental study. Sci Rep 201.

7. Brandom B, Larach MG, Chen MA, Young M (2011) Complications Associated with the Administration of Dantrolene 1987 to 2006: A Report from the North American Malignant Hyperthermia Registry of the Malignant Hyperthermia Associate of the United States. Anesth Analg 112: 1115-1123.

8. Javed M, Bogdancov A (2010) Oral dantrolene and severe respiratory failure in a patient with chronic spinal cord injury. Anaesthesia 65: 855-856.

9. Flewellen EH, Nelson TE, Jones WP, Arens JF, Wanger DL (1983) Dantrolene Dose Response in Awake Man: Implicatoins for Management of Malignan Hyperthermia. Anesthesiology 59: 275-280.

10. Langeron O, Coirault C, Fratea S, Coriate P, Riou B (1998) Effects of Dantrolene on the diaphragm muscle of normal and mypathic hamster. $\mathrm{Br} J$ Anaesth 81: 553-555.

11. Langeron O, Coirault C, Fratea S, Orliaguet G, Coriate P, et al. (1999) Effects of Dantrolene on the Contraction, Relaxation, and Energetics of the Diaphragm Muscle. Anesth Analg 89: 466-471. 